\title{
DEFECT LUMINESCENCE IN HEAVILY Mg DOPED GaN
}

\author{
M. A. Reshchikov*, G.-C. Yi, and B. W. Wessels \\ Department of Materials Science and Engineering and Materials Research Center \\ Northwestern University, Evanston, IL, 60208
}

Cite this article as: MRS Internet J. Nitride Semicond. Res. 4S1, G11.8 (1999)

\begin{abstract}
Behavior of the photoluminescence band at about $2.8 \mathrm{eV}$ in heavily $\mathrm{Mg}$ doped $\mathrm{GaN}$ has been studied at different temperatures and excitation intensities. The $2.8 \mathrm{eV}$ band is attributed to donor-acceptor transitions involving a $\mathrm{Mg}$ acceptor. The large blue shift of the band with increasing excitation intensity is explained by variation in the contribution of close and distant pairs to the luminescence. The red shift of the band with increasing temperature under high excitation intensity conditions results from thermal release of carriers from close pairs. The thermal activation energy of the deep donor, about $0.4 \mathrm{eV}$, is determined from the quenching of the $2.8 \mathrm{eV}$ luminescence band at high temperatures.
\end{abstract}

\section{INTRODUCTION}

High concentrations of magnesium acceptors are required to obtain conductive p-type $\mathrm{GaN}$ because of its large activation energy. In heavily $\mathrm{Mg}$ doped $\mathrm{GaN}$ a broad photoluminescence (PL) band around 2.6-2.95 eV (referred hereafter as the $2.8 \mathrm{eV}$ band) dominates the PL spectrum ${ }^{1-11}$. The nature of the defect responsible for this band and even the type of optical transitions involved remain unclear. The most widely accepted model is that the PL is due to an optical transition from the conduction band to a deep defect with a level at about $0.5-0.55 \mathrm{eV}$ above the valence band ${ }^{2-6}$. Recently, it was proposed that the $2.8 \mathrm{eV}$ PL band involves an optical transition from a deep donor to the shallow $\mathrm{Mg}$ acceptor ${ }^{7}$. Up to now there is no definite evidence supporting one particular model. The main distinguishing feature of the 2.8 $\mathrm{eV}$ band, a large blue shift with increasing excitation intensity, may be explained by several models ${ }^{5-8}$. In the donor-acceptor pair (DAP) model, the shift is attributed to saturation of emission from distant DAP due to their longer lifetime ${ }^{7,8}$. Alternatively, formation of impurity bands ${ }^{5,6}$ or potential fluctuations ${ }^{6}$ have been considered as possible reasons for the observed shift.

Indeed, large potential fluctuations due to randomly distributed charged impurities are expected in heavily doped semiconductors ${ }^{12}$. Free carriers in compensated material are localized in potential wells and optical transitions have many similar features to that of the DAP type transitions ${ }^{13}$. The similarities in behavior of the DAP emission and the band-assisted transitions in heavily doped semiconductor with potential wells complicate assignment of PL bands.

In this work we investigated the $2.8 \mathrm{eV}$ PL band in heavily doped GaN at different temperatures and excitation intensities. The results of the study support the deep donor-acceptor pair assignment of this PL. The large measured shifts of the $2.8 \mathrm{eV}$ band with variation of temperature or excitation intensity originate from varying contribution of emission from close and distant pairs. 


\section{EXPERIMENTAL RESULTS}

The Mg-doped GaN epilayers were grown on c-plane sapphire by metal-organic vapor phase epitaxy. The samples are either semi-insulating or p-type with resistivity as low as $3 \Omega \cdot \mathrm{cm}$ at room temperature. PL was excited by cw He-Cd laser $(325 \mathrm{~nm})$ and analyzed by Spex grating monochromator with Hamamatsu photomultiplier tube. Excitation power density $\left(\mathrm{P}_{\mathrm{exc}}\right)$ was varied over the range $10^{-5}-27 \mathrm{~W} / \mathrm{cm}^{2}$ by means of neutral density filters. The sample temperature was varied from 13 to $380 \mathrm{~K}$ using a closed cycle helium cryostat.

A broad band with a peak near $2.8 \mathrm{eV}$ has been observed in the PL spectra of the studied samples. In some samples another PL band with a maximum near $3.2 \mathrm{eV}$ was noted as a shoulder or separate peak. The position of the $2.8 \mathrm{eV}$ band depended strongly on excitation intensity (Fig. 1). At low temperature the total blue shift of the band with increasing excitation power amounted to $0.23 \mathrm{eV}$. The peak position shifted largely at high excitation density and was nearly independent of excitation rate in the low excitation limit. At room temperature the PL band shifted about $0.10 \mathrm{eV}$ over the same excitation intensity range (Fig. 1). The shape of the band remained almost independent of excitation intensity for the samples with small overlap with the $3.2 \mathrm{eV}$ band. A typical change in the PL spectrum is shown in Fig. 2. We attribute the observed dependence of the PL spectrum with excitation to changes in contributions of emission from close and distant pairs in the case of deep DAP transitions. The DAP transition rate for a pair with a separation $R$ has the approximate form ${ }^{13,14}$

$$
W(R)=W_{0} \exp \left(-\frac{2 R}{a_{B}}\right)
$$

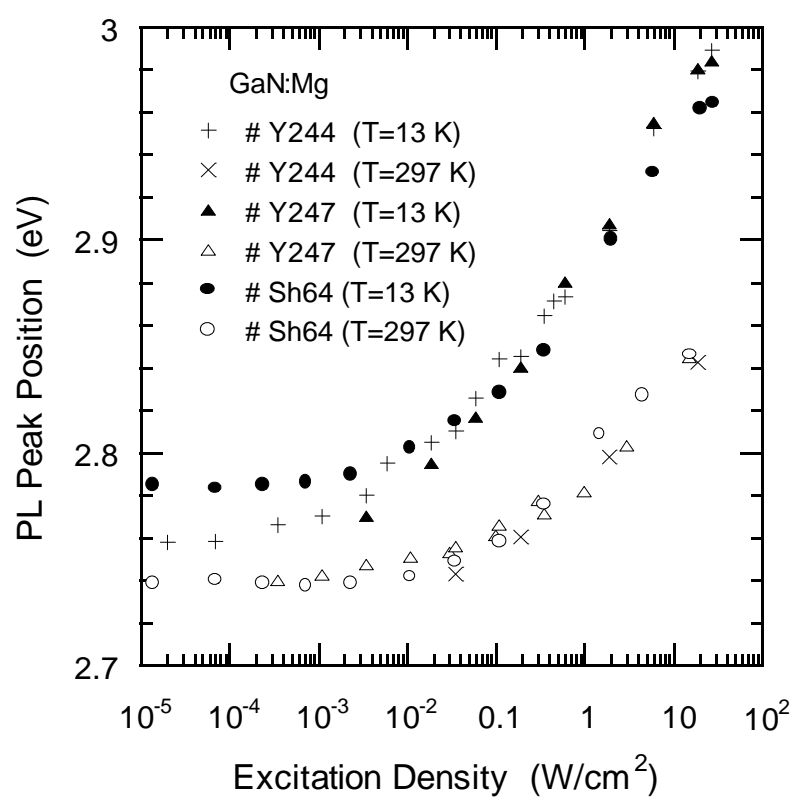

Fig. 1. Dependence of the $2.8 \mathrm{eV}$ PL peak position on excitation power density at 13 and $297 \mathrm{~K}$ for three GaN:Mg samples.

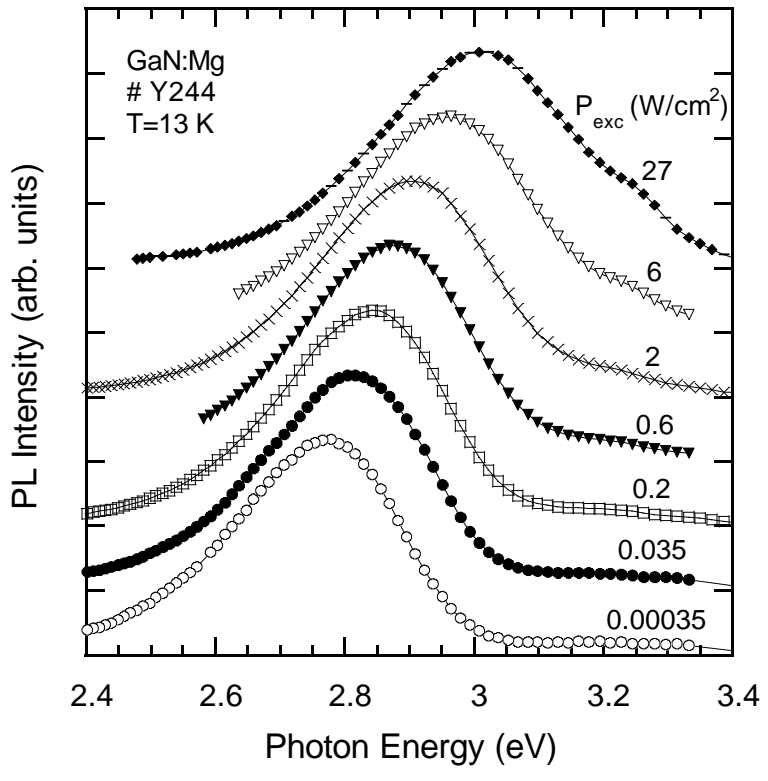

Fig. 2. PL spectra of the semiinsulating GaN:Mg sample \# Y244 at different excitation intensities. 
where $a_{B}$ is the largest Bohr radius for the bound carriers. The radiative lifetime increases exponentially with increasing $R$ according to Eq. (1). At high excitation intensity, PL from distant pairs saturates due to their longer lifetime and transitions between close pairs dominate. The PL energy corresponding to a transition between a pair with separation $R$ is given by

$$
E_{P L}(R)=E_{g}-\left(E_{A}+E_{D}\right)+\frac{e^{2}}{\varepsilon R}
$$

where $E_{g}$ is the band gap, $E_{A}$ and $E_{D}$ are the acceptor and donor binding energies, $e$ is the electronic charge, and $\varepsilon$ is the low frequency dielectric constant. The last term is due to the Coulomb interaction between charged donor and acceptor. As a result of strong Coulomb interaction between close pairs, the PL band blue-shifts with increasing excitation intensity. Saturation of the band shift in the low excitation limit may be explained by the fact that transitions between pairs with more than average separation are highly unlikely because of their small fraction and the low probability of such transitions.

For a high excitation intensity the $2.8 \mathrm{eV}$ band red-shifted significantly with increasing temperature whereas at low excitation intensity its position remained almost unchanged up to 100 - $200 \mathrm{~K}$ (Fig. 3). This behavior also may be explained by the DAP nature of this band. The Coulomb interaction between close pairs may be very strong ${ }^{14}$ and the resultant ionization energy will decrease sufficiently enough for thermal release of the trapped carriers. As a result, the high-energy portion of the spectrum begins quenching at lower temperatures and the PL band undergoes a red shift. However at a low excitation rate, emission from comparatively distant pairs dominates and the red shift is reduced. This effect has been previously observed in Si doped with donors and acceptors having similar activation energies ${ }^{15}$. The observed temperature-induced red shift is just the reverse of the well-known blue shift for DAP emission for shallow donors and acceptors ${ }^{13}$. In the latter case the blue shift is due to thermal escape of electrons from the shallow donor which is more favorable for the long-lived distant pairs contributing to the low-energy part of a spectrum. The observed red shift of the $2.8 \mathrm{eV}$ band with increase in temperature can not be attributed to potential fluctuations since the red shift caused by the potential fluctuations should increase with a decrease of excitation rate ${ }^{13}$.

The temperature dependence of the PL intensity for the $2.8 \mathrm{eV}$ band is shown in Fig. 4. The $2.8 \mathrm{eV}$ PL band quenches at temperatures above $200-250 \mathrm{~K}$ with an activation energy $E_{D}$ of about 0.3-0.4 eV, observed for all samples. The quenching is attributed to thermal release of electrons from deep donor to the conduction band. In the inset of Fig. 4 the calculated dependence of the PL intensity is shown using the following expression

$$
I(T)=I_{0}\left(1+A \exp \left(-\frac{E_{D}}{k T}\right)\right)^{-1}
$$

where $A$ is a constant, assumed to be temperature independent for simplicity. We attribute the discrepancy between calculated curve and the experimental data to the distribution of donor energies due to the Coulomb interaction with the charged acceptors. The higher the excitation intensity, the higher the contribution of the close pairs characterized by the stronger Coulomb interaction. Thermalization of electrons to the conduction band from the donors of these close pairs results in the beginning of thermal quenching of PL at lower temperatures, see the inset of Fig. 4.

The lifetime of the $2.8 \mathrm{eV}$ PL has been estimated to be $10^{-5} \mathrm{sec}$ at low temperature from the time-resolved measurements. The decay of the PL is non-exponential. These results are also consistent with the DAP model for the $2.8 \mathrm{eV}$ PL band. 


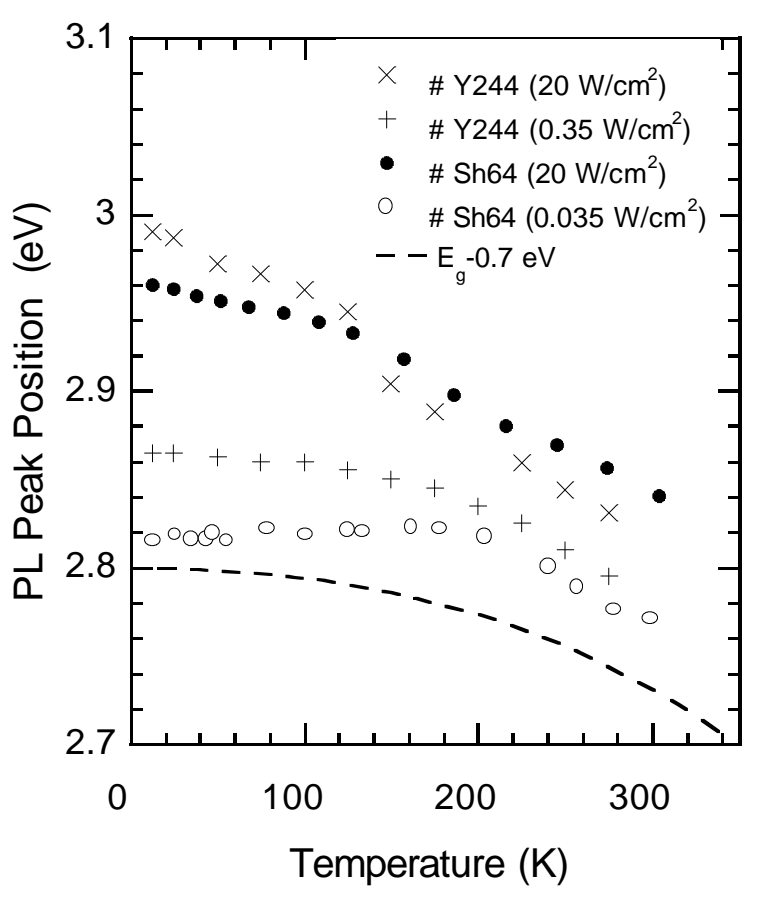

Fig. 3. Variation of the $2.8 \mathrm{eV} \mathrm{PL}$ peak position with temperature. Variation of the gap width is shown shifted by $0.7 \mathrm{eV}$ for convenience.

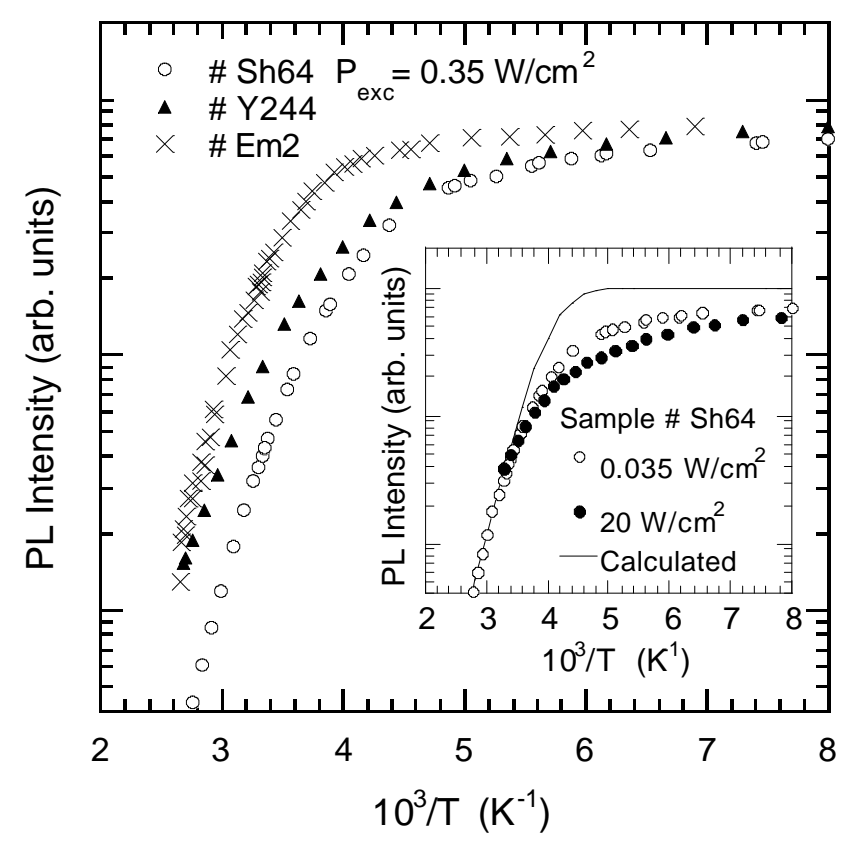

Fig. 4. Intensity of the $2.8 \mathrm{eV}$ PL band versus reverse temperature. The inset shows the $I\left(T^{-1}\right)$ dependence at low and high excitation intensities in comparison with the calculated from Eq. (3) dependence $\left(A=1.5 \times 10^{7} ; E_{D}=0.35 \mathrm{eV}\right)$.

\section{DISCUSSION}

The 2.8 eV PL band appears at high concentrations of $\mathrm{Mg}\left(10^{19}-10^{20} \mathrm{~cm}^{-3}\right)^{1,2,6,7,10}$ which correspond to a mean separation between impurities of about 15-30 A. The close location of pair components results in a high probability for DAP type transitions in spite of the strong localization of the wave-functions for electron and hole bound to the deep defects. The Coulomb interaction energy for an average separation between acceptor and donor is estimated to be $0.1-0.15 \mathrm{eV}$ by taking $R=10-15 \AA$ (corresponds to acceptor concentration of $\sim 10^{19} \mathrm{~cm}^{-3}$ ) in Eq. (2). Supposing that saturation of the PL band peak position in the low excitation limit (at 2.75-2.78 eV in Fig. 1) corresponds to transitions between DAP having an average separation, and taking $E_{g}=3.5 \mathrm{eV}$ and $E_{A}=0.2 \mathrm{eV}$, we calculated $E_{D}=0.6-0.7 \mathrm{eV}$ for the isolated deep donor. The actual position of the donor level in heavily doped $\mathrm{GaN}$ depends essentially on the location of the nearest charged acceptor, shown schematically in Fig. 5.

The potential fluctuations, expected for heavily doped semiconductors ${ }^{12}$, should not strongly affect the deep DAP emission. Indeed, the higher the magnitude of the potential fluctuation, the larger its size ${ }^{12}$, and optical transition between defects separated by about 10-15 $\AA$ may be considered as almost vertical in the scale of the long-range fluctuations. 


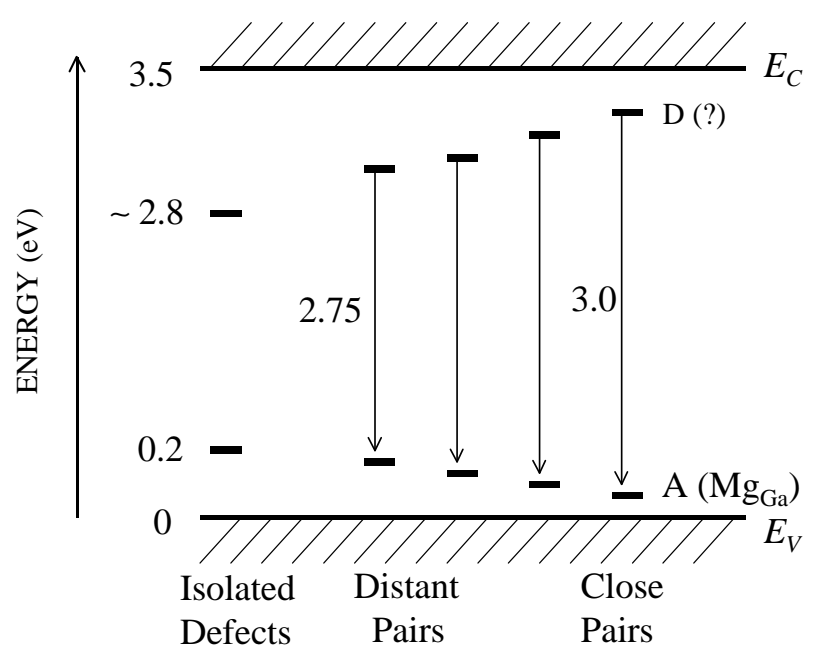

Fig. 5. Schematic diagram showing optical transitions in heavily $\mathrm{Mg}$ doped $\mathrm{GaN}$

The values of radiative lifetime for the $2.8 \mathrm{eV}$ band found in this study (about $10 \mu \mathrm{sec}$ ) and by Seitz et al. ${ }^{11}$ (about $0.2 \mathrm{msec}$ ) are consistent with DAP emission. Similar lifetimes were observed for the deep DAP in ZnSe (about $0.1 \mathrm{msec}$ ) ${ }^{14}$.

The question of the nature of donor responsible for the compensation of $\mathrm{Mg}$ in $\mathrm{GaN}$ is open. The nitrogen vacancy $\left(\mathrm{V}_{\mathrm{N}}\right)$, which has a low formation energy in p-type $\mathrm{GaN}$, is one possibility. However the vacancy is expected to have the $+/ 3+$ energy level not far from the valence band ${ }^{16}$. It is possible that the donor is formed from a complex defect. Kaufmann et al. suggested that $\mathrm{Mg}_{\mathrm{Ga}}$ and mobile $\mathrm{V}_{\mathrm{N}}$ form a complex $\mathrm{Mg}_{\mathrm{Ga}} \mathrm{V}_{\mathrm{N}}$ that acts as a donor ${ }^{7}$. The identification of this defect requires further investigations.

\section{CONCLUSIONS}

A large blue shift of the $2.8 \mathrm{eV}$ PL band with increasing excitation intensity has been observed in $\mathrm{Mg}$ doped $\mathrm{GaN}$. This effect, as well as the observed red shift of the band with increasing temperature at high excitation rate, is explained by variation in contributions of emission from close and distant pairs to the PL band. The $2.8 \mathrm{eV}$ band is attributed to transitions from the compensating deep donor to the shallow $\mathrm{Mg}$ acceptor. Thermal quenching of the $2.8 \mathrm{eV}$ band begins at $\mathrm{T}>200 \mathrm{~K}$ with an apparent activation energy of about $0.4 \mathrm{eV}$. The observed quenching is attributed to the thermalization of trapped electrons from the deep donor state to the conduction band.

\section{ACKNOLEDGEMENTS}

The authors thank F. Shahedipour at the Electronic and Photonic Materials Group and Ian Ferguson at EMCORE for providing p-type GaN samples. This work was supported by the National Science Foundation GOALI Program, under grant number ECS-9705134. 


\section{REFERENCES}

* On leave from A. F. Ioffe Physico-Technical Institute of Russian Academy of Sciences, St. Petersburg, Russia.

1. H. Amano, M. Kitoh, K. Hiramatsu, I. Akasaki, J. Electrochem. Soc., 137, 1639 (1990).

2. A. K. Viswanath, E. Shin, J. I. Lee, S. Yu, D. Kim, B. Kim, Y. Choi and C.-H. Hong, J.Appl. Phys. 83, 2272 (1998).

3. H. P. Maruska, D. A. Stevenson and J.I. Pankove, Appl. Phys. Lett. 22, 303 (1973).

4. S. Nakamura, N. Iwasa, M. Senoh, and T. Mukai, Jpn. J. Appl. Phys. 31, Pt. 1, 1258 (1992).

5. M. Smith, G. D. Chen, J. Y. Lin, H. X. Jiang, A. Salvador, B. N. Sverdlov, A. Botchkarev, H. Morkoc, and B. Goldenberg, Appl. Phys. Lett. 68, 1883 (1996).

6. E. Oh, H. Park and Y. Park, Appl. Phys. Lett 72, 70 (1998).

7. U. Kaufmann, M. Kunzer, M. Maier, H. Obloh, A. Ramakrishnan, B. Santic, and P. Schlotter, Appl. Phys. Lett. 72, 1326 (1998).

8. L. Eckey, U. Von Gfug, J. Holst, A. Hoffmann, B. Schineller, K. Heime, M. Heuken, O. Schön, and R. Beccard, J. Cryst. Growth 189/190, 523 (1998).

9. J. M. Myoung, K. H. Shim, C. Kim, O. Gluschenkov, K. Kim, S. Kim, D. A. Turnbull, and S.G. Bishop, Appl. Phys. Lett. 69, 2722 (1996).

10. W. Götz, N. M. Johnson, J. Walker, D. P. Bour, and R. A. Street, Appl. Phys. Lett. 68, 667 (1996).

11. R. Seitz, C. Gaspar, T. Monteiro, E. Pereira, M. Leroux, B. Beaumont, and P. Gibart, Materials Science Forum 258-263, 1155 (Trans. Tech. Publications, Switzerland, 1997).

12. B. I. Shklovskii and A. L. Efros, Electronic Properties of Doped Semiconductors (Springer, Berlin, 1984), pp. 53-73, 253-313.

13. A. P. Levanyuk, V. V. Osipov, Usp. Fiz. Nauk 133, 427 (1981) [Sov. Phys. Usp. 24, 187 (1981)].

14. F. C. Rong, W. A. Barry, J. F. Donegan, and. G. D. Watkins, Phys. Rev. B 54, 7779 (1996); W. A. Barry and G. D. Watkins, ibid., 7789 (1996).

15. A. S. Kaminskii and Ya. E. Pokrovskii, Fiz. Tekh. Poluprovodn. 3, 1766 (1969) [Sov. Phys. Semicond. 3, 1496 (1970)].

16. C. G. Van de Walle, C. Stampfl and J. Neugebauer, J. Cryst. Growth 189/190, 505 (1998). 